\title{
ASSOCIAÇÃO ENTRE CASOS DE AIDS EM MENORES DE CINCO ANOS E COBERTURA DA ATENÇÃO BÁSICA
}

\section{ASSOCIATION BETWEEN AIDS CASES IN CHILDREN UNDER FIVE AND COVERAGE OF PRIMARY CARE}

\section{ASOCIACIÓN ENTRE CASOS DE SIDA EN MENORES DE CINCO AÑOS Y COBERTURA DE ATENCIÓN PRIMARIA}

Samara Eliane Rabelo Suplici ${ }^{1}$, Sabrina da Silva de Souza², Ana Cláudia da Cunha ${ }^{3}$, Katheri Maris Zamprogna ${ }^{4}$, Dione Lúcia Prim Laurindo 5

\section{RESUMO}

Objetivo: Avaliar o número de casos novos de AIDS em menores de cinco anos de idade e sua associação com a cobertura populacional pelas equipes de Atenção Básica. Método: Estudo transversal que utilizou dados secundários do banco de dados oficiais do Estado de Santa Catarina. Os indicadores número de casos novos de AIDS em menores de cinco anos de idade e cobertura populacional pelas equipes de Atenção Básica foram coletados. Estatísticas descritivas foram calculadas como médias e desviospadrão e realizado o coeficiente de correlação de postos de Spearman no software SPSS 25. Resultados: O número de casos novos de AIDS, em menores de cinco anos de idade, aumentou no período estudado e teve correlação significativa e inversa com a cobertura populacional pelas equipes de atenção básica. Conclusão: A correlação encontrada evidencia a importância da cobertura populacional pelas equipes de Atenção Básica no controle da cadeia de transmissão da AIDS.

Descritores: Atenção Primária à Saúde; Saúde da Família; Cuidados de Enfermagem; Síndrome da Imunodeficiência Adquirida; HIV.

\section{ABSTRACT}

Objective: To evaluate the number of new AIDS cases in children under five years old and their association with the population coverage by Primary Care teams. Method: A cross-sectional study that used secondary data from the official database of the State of Santa Catarina. The indicators 'number of new AIDS cases in children under five' and 'population coverage by Primary Care teams' were collected. Descriptive statistics were calculated, such as means and standard deviations, and the Spearman rank correlation coefficient was calculated using the SPSS 25 software. Results: The number of new AIDS cases in children under five increased and was conversely correlated with the population coverage provided by primary care teams. Conclusion: The correlation found shows the importance of population coverage by Primary Care teams in controlling the disease transmission chain.

Descriptors: Primary Health Care; Family Health; Nursing Care; Acquired Immunodeficiency Syndrome; HIV.

\section{RESUMEN}

Objetivo: Evaluar el número de casos nuevos de SIDA en niños menores de cinco años y su asociación con la cobertura de población por parte de los equipos de Atención Primaria. Método: Estudio transversal que utilizó datos secundarios de la base de datos oficial del Estado de Santa Catarina. Se recolectó el número de indicadores de nuevos casos de SIDA en niños menores de cinco años y la cobertura de la población por parte de los equipos de atención primaria. Las estadísticas descriptivas se calcularon como medias y desviaciones estándar y el coeficiente de correlación de rango de Spearman se calculó utilizando el software SPSS 25. Resultados: El número de nuevos casos de SIDA en niños menores de cinco años aumentó y se correlacionó a la inversa con la cobertura poblacional de los equipos de atención primaria. Conclusión: La correlación encontrada muestra la importancia de la cobertura de la población por parte de los equipos de atención primaria en el control de la cadena de transmisión de la enfermedad.

Descriptores: Atención Primaria de Salud; Salud de la Familia; Atención de Enfermería; Síndrome dade Inmunodeficiencia Adquirida; VIH.

${ }^{1}$ Graduada em Enfermagem. Doutora em Enfermagem pela UFSC. Membro do Nucron-UFSC. ${ }^{2}$ Graduada em Enfermagem. Doutora em Enfermagem pela UFSC. Enfermeira do HU/UFSC e Secretaria Municipal de São José/SC. ${ }^{3}$ Graduada em Enfermagem. Especialista em Enfermagem na Saúde da Família pela UFSC. Intituição de origem: Secretaria Municipal de São José/SC. ${ }^{4}$ Graduada em Enfermagem. Doutora em Enfermagem pela UFSC. Enfermeira da Secretaria Municipal de São José/SC. ${ }^{5}$ Graduada em Enfermagem. Especialista em Enfermagem na Saúde da Família pela UFSC. Intituição de origem: Secretaria Municipal de São José/SC.

Como citar este artigo:

Suplici SER, Souza SS, Cunha AC, et al. Associação entre casos de aids em menores de cinco anos e cobertura da atenção básica. Revista de Enfermagem do Centro-Oeste Mineiro. 2020;10:e3908. [Access___]; Available in:___ DOI: http://doi.org/10.19175/recom.v10i0.3908 


\section{INTRODUÇÃO}

A Síndrome da Imunodeficiência Adquirida (AIDS), causada pelo vírus da Imunodeficiência Humana (HIV), foi constatada pelo Centers for Disease Control and Prevention (CDC) de Atlanta EUA, em 1981. Nessas mais de três décadas, desde a sua descoberta, a infecção pelo HIV e a AIDS se transformaram em uma epidemia global e inúmeros avanços científicos foram alcançados, incluindo a identificação dos mecanismos da doença e a introdução da terapia antirretroviral (TARV). Apesar dessas descobertas científicas terem resultado em melhores cuidados de saúde e aumentado a longevidade das pessoas infectadas, a infecção pelo HIV e AIDS ainda representam um desafio para os sistemas de saúde no mundo ${ }^{(1-2)}$.

No Brasil, a infecção pelo HIV e a AIDS fazem parte da Lista Nacional de Notificação Compulsória de doenças, sendo que a AIDS é de notificação compulsória, desde 1986 e a infecção pelo HIV é de notificação compulsória desde 2014 ${ }^{(3)}$. De 1980 a 2019, foram identificados 966.058 casos de AIDS no Brasil. O país tem registrado, anualmente, uma média de 39 mil novos casos nos últimos cinco anos ${ }^{(2)}$. is sep.

No entanto, a taxa de deteç̧ão dessa doença vem caindo, no Brasil, nos últimos anos. Desde o ano de 2012, observa-se uma diminuição nessa taxa, configurando um decréscimo de $16,8 \%$. Essa redução na taxa de deteç̧ão tem sido mais acentuada, desde a recomendação do tratamento para todos, implementada em dezembro de 2013. A medida trouxe mais acesso ao tratamento e aumento de diagnósticos. Com isso, nos últimos cinco anos, a tendência de queda na taxa de AIDS foi maior ${ }^{(2)}$.

A despeito disso, a infecção por HIV cresceu entre as gestantes. Nessa população, houve um aumento de $38,1 \%$ na taxa de detecção de HIV, em um período de dez anos. Esse aumento pode ser explicado, em parte, pela ampliação do diagnóstico no pré-natal e a consequente prevenção da transmissão vertical do $\mathrm{HIV}^{(2)}$.

Tendo em vista que a gestante infectada pode transmitir o vírus para o feto, durante a gestação/parto ou para o recém-nascido, durante a amamentação, a transmissão vertical do HIV ainda é um desafio de saúde pública para diversos países. O Brasil firmou um compromisso de eliminar a transmissão vertical do HIV que é uma das seis prioridades do Departamento de Doenças de Condições Crônicas e Infecções Sexualmente Transmissíveis (DCCI) da Secretaria de Vigilância em Saúde do Ministério da Saúde brasileiro ${ }^{(4-5)}$.

A efetividade das políticas de prevenção da transmissão vertical do HIV pode ser mensurada pelo número de casos novos dessa doença, em menores de 5 anos de idade. Esse número tem sido utilizado como indicador para o monitoramento da transmissão vertical do HIV e, por isso, esse dado faz parte da pactuação interfederativa de indicadores para o período 2017-2021, relacionados a prioridades nacionais em saúde ${ }^{(4)}$.

De 2014 a 2018, houve redução de 26,9\% na taxa de detecção de AIDS em menores de 5 anos de idade, passando de 2,6 em 2014 (386 casos) para 1,9 casos (265 casos) por $100 \mathrm{mil}$ habitantes em 2018. Pressupõe- se que essa tendência de declínio esteja associada ao tratamento adequado das gestantes infectadas pelo HIV, o que evita a transmissão vertical e, consequentemente, diminui o número de casos em crianças ${ }^{(2)}$. Ações realizadas pelo sistema de saúde que vão desde o acesso ao atendimento pré-natal e testagem para o HIV, até o seguimento da criança exposta, podem garantir o sucesso na redução da transmissão vertical do $\operatorname{HIV}^{(5)}$.

Nesse sentido, a atuação da Atenção Primária à Saúde (APS) no cuidado à gestante merece destaque. No Brasil, desde os anos de 1990, nota-se uma cobertura crescente da assistência pré-natal no âmbito da APS, alcançando valores superiores a $90 \%$ em todas as regiões do país e em mulheres com diferentes características demográficas, sociais e reprodutivas. Sabe-se que o cuidado pré-natal é fundamental para o bem-estar materno e fetal, e pode contribuir para a prevenção da transmissão vertical pelo HIV ${ }^{(6-7)}$.

A literatura aponta que uma redução de casos de HIV/AIDS pode ser alcançada com a promoção de ações educativas, disponibilização de testes rápidos de forma regular e oferta de serviços que garantam o tratamento das pessoas infectadas, sendo que, essas ações podem ser alcançadas, por meio de maior acesso à APS ${ }^{(7)}$. $\mathrm{Na}$ última década, maior acesso à APS contribuiu, de algum modo, para um avanço, na saúde das pessoas infectadas pelo HIV ${ }^{(5-7)}$. O acesso às ações e serviços da APS pode ser mensurado por meio da cobertura populacional pelas equipes de Atenção Básica que considera a centralidade da APS como ordenadora do cuidado, consolidandose num indicador fundamental, o qual também foi pactuado ${ }^{(4)}$. No entanto, existe uma lacuna nas 
publicações sobre a influência da cobertura populacional pelas equipes de Atenção Básica na redução da incidência de AIDS, em menores de cinco anos. Desse modo, tem-se como questão: o número de casos novos dessa doença, em menores de cinco anos de idade, está associado à cobertura populacional pelas equipes de Atenção Básica?

A hipótese é que exista uma associação entre o número de casos novos de AIDS em menores de cinco anos de idade e a cobertura populacional pelas equipes de Atenção Básica, ou seja, uma redução no número de casos em menores de cinco anos está associada a uma maior cobertura populacional pelas equipes de Atenção Básica.

Assim, objetivou-se, neste estudo, avaliar o número de casos novos de AIDS em menores de cinco anos de idade e sua associação com a cobertura populacional pelas equipes de Atenção Básica.

\section{MÉTODOS}

Estudo ecológico transversal realizado com base em dados secundários da Pactuação Interfederativa 2017 - 2021. Os dados secundários foram obtidos no banco de dados oficiais da Diretoria de Vigilância Epidemiológica do Estado de Santa Catarina (DIVE/SC) ${ }^{(8)}$. Esses dados são de acesso público. Para este estudo, foram extraídos do banco de dados os indicadores: número de casos novos de AIDS em menores de cinco anos de idade e a cobertura populacional pelas equipes de Atenção Básica. O período estudado foram os anos 2017 e 2018.

A variável dependente foi o número de casos novos de AIDS em menores de cinco anos de idade. Essa variável considera o número de casos novos da doença, na população de menores de 5 anos de idade, residente em determinado local, no ano considerado, medindo o risco de ocorrência de casos novos, nessa população. A unidade de medida é expressa em número absoluto ${ }^{(4)}$.

A variável independente foi a cobertura populacional pelas equipes de Atenção Básica. Essa variável considera o valor de 3.450 indivíduos, cobertos por equipe de Saúde da Família, e 3.000 indivíduos cobertos pelas equipes de Atenção Básica parametrizadas e equipes equivalentes. Desse modo, o método de cálculo tem como numerador: o número de equipes de Saúde da Família x 3.450 + (número de equipes de Atenção Básica parametrizadas e equipes equivalentes) $x$ 3.000 em determinado local e período. O denominador é a estimativa populacional do ano anterior. A unidade de medida é expressa em porcentagem. Essa variável é utilizada para o monitoramento do acesso aos serviços da APS, com vistas ao fortalecimento do planejamento do Sistema Único de Saúde (SUS) e sua capacidade de resolução(4).

Todos os dados coletados foram inseridos em uma planilha no Excel $2016^{\circledast}$ (Microsoft Office) com dupla digitação. Posteriormente, foram exportados para os softwares Statistical Package for the Social Sciences (SPSS), versão $25.0 \mathrm{da}$ plataforma de software estatístico IBM e R - um ambiente com um conjunto integrado de recursos de software para tratamento de dados, cálculos e exibição gráfica ${ }^{(9)}$, onde as análises foram realizadas. Foram conduzidas análises descritivas, por meio de medidas das médias, desvios-padrão, mínimos e máximos para os indicadores do estudo. Para testar a hipótese de associação entre os indicadores estudados foi proposta uma matriz de correlação a partir do cálculo do coeficiente de correlação de postos de Spearman. Todos os testes levaram em consideração um $\alpha$ bidirecional de 0.05 e um intervalo de confiança (IC) de $95 \%$. Valor de $p$ menor que 0,05 foi utilizado como ponto de corte para significância estatística.

$\mathrm{Na}$ matriz de correlação de Spearman, apresentada neste trabalho, a variável número de casos novos de AIDS em menores de cinco anos de idade será apresentada como V12 e a variável Cobertura populacional pelas equipes de Atenção Básica será apresentada como V23. Isso porque, este estudo é parte de um macroprojeto que avaliou a cobertura populacional pelas equipes de Atenção Básica nos municípios de Santa Catarina e o alcance dos indicadores, de acordo com os dados da pactuação interfederativa 2017-2021. Nesse macroprojeto, cada um dos 23 indicadores da pactuação interfederativa foi tratado como uma variável de análise, sendo identificados pela letra V (variável) seguida de uma numeração arábica (1 a 23).

Quanto aos aspectos éticos, neste estudo, utilizaram-se dados de sistemas oficiais de informações de saúde de domínio público, de modo que foi dispensado de apreciação por comitê de ética. A Resolução no 510, de 04/07/2016, do Conselho Nacional de Saúde (CNS), em seu parágrafo único, determina que não precisará ser avaliada pelo Comitê de Ética e Pesquisa, as pesquisas que usam informações de domínio público. 


\section{RESULTADOS E DISCUSSÃO}

Ficou evidenciado que o número de casos novos de AIDS, em menores de cinco anos de idade, aumentou, em Santa Catarina, entre os anos de 2017 e 2018. $O$ estado apresentou 3 casos novos em 2017 e 5 casos novos em 2018. A cobertura populacional pelas equipes de Atenção Básica, por sua vez, diminuiu no período estudado, tendo médias de 82,8 em 2017 e 82,4 em 2018, conforme apresentado na Tabela 1.

Tabela 1 - Número de casos novos de AIDS em menores de cinco anos de idade e cobertura populacional pelas equipes de Atenção Básica, nos anos de 2017 e 2018. Santa Catarina, Brasil, 2019.

\begin{tabular}{|c|c|c|c|c|c|c|c|c|}
\hline \multirow[b]{2}{*}{ Indicador } & \multicolumn{4}{|c|}{2017} & \multicolumn{4}{|c|}{2018} \\
\hline & Média & $\begin{array}{l}\text { Desvio } \\
\text { Padrão }\end{array}$ & Mínimo & Máximo & Média & $\begin{array}{l}\text { Desvio } \\
\text { Padrão }\end{array}$ & Mínimo & Máximo \\
\hline $\begin{array}{l}\text { Número de casos novos de AIDS em menores de cinco } \\
\text { anos de idade* }\end{array}$ & 0 & 0 & 0 & 3 & 0 & 0 & 0 & 5 \\
\hline Cobertura populacional pelas equipes de Atenção Básica† & 82.8 & 25.3 & 0 & 100 & 82.4 & 25.5 & 0 & 100 \\
\hline
\end{tabular}

Fonte: Diretoria de Vigilância Epidemiológica (DIVE/SC).

* A Meta pactuada pelo estado foi 2 casos novos para 2017 e 6 casos novos para $2018^{(10)}$.

† A meta pactuada pelo estado foi $88,50 \%$ para 2017 e $88,16 \%$ para $2018^{(10)}$.

Quanto ao número de casos novos, em menores de cinco anos de idade, foi evidenciado ainda que no ano de 2017, 292 municípios (98,86\%) não apresentaram novos casos e três municípios apresentaram registros de novos casos. Cada um desses municípios apresentou 70,33\%, $74,45 \%$ e $77,77 \%$ de cobertura populacional pelas equipes de Atenção Básica. No ano de 2018, em 291 municípios $(98,48 \%)$ o número de casos novos, em menores de cinco anos de idade, foi igual a zero e quatro municípios registraram casos novos, sendo que três municípios apresentaram um caso e um município apresentou dois casos. Essas quatro cidades apresentaram 100\%, 87,03\%, $87,07 \%$ e $91,65 \%$ de cobertura populacional, pelas equipes de Atenção Básica.
No que se refere à cobertura populacional pelas equipes de Atenção Básica, os resultados mostraram ainda que, em 2017, 86,78\% dos municípios tiveram a cobertura superior à meta pactuada e $13,22 \%$ dos municípios catarinenses tiveram cobertura inferior à meta. Em 2018, 87,12\% apresentaram cobertura superior à meta e $12,88 \%$ apresentaram cobertura inferior à meta pactuada $^{(10)}$.

A matriz de correlação entre os vários indicadores da pactuação interfederativa ${ }^{(4)}$ no estado de Santa Catarina, nos anos de 2017 e 2018, apresentada na Figura 1, mostra que o número de casos novos de AIDS em menores de cinco anos de idade (apresentado em V12) está, inversamente, correlacionado à cobertura populacional pelas equipes de Atenção Básica (apresentado em V23).

Figura 1 - Matriz de correlação dos indicadores número de casos novos de AIDS em menores de cinco anos de idade (V12) e cobertura populacional pelas equipes de Atenção Básica (V23), nos anos de 2017 e 2018. Santa Catarina, Brasil, 2019.
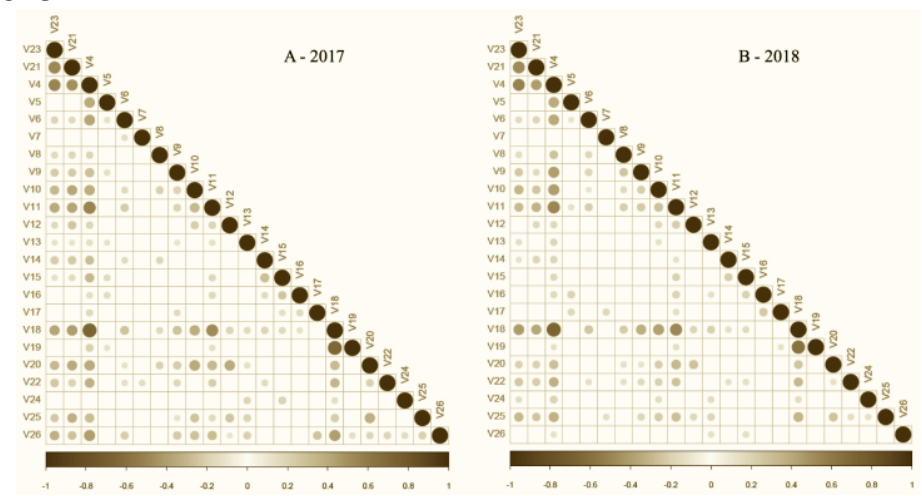

Teste de correlação de Spearman (na Figura 1 quanto mais forte a cor mais bem correlacionado é o indicador).

Fonte: elaborado pelos autores, 2020.

Os resultados do presente estudo evidenciaram um aumento do número de casos novos de AIDS, em menores de cinco anos de idade, entre os anos de 2017 e 2018 , no estado de
Santa Catarina. Esse dado difere da tendência atual, na maioria dos estados brasileiros, pois, de modo geral, verifica-se significativa tendência de queda desse indicador no país. A literatura aponta 
que o número de casos da doença, em menores de cinco anos de idade vem diminuindo, no Brasil, embora existam muitas diferenças regionais. Supõe-se que essas diferenças sejam reflexo das desigualdades na rede instalada de serviços de saúde no país ${ }^{(11)}$.

Apesar do aumento registrado no número de casos novos, em menores de cinco anos de idade, no período estudado, historicamente, ao se analisar os demais anos, o estado de Santa Catarina vem apresentando redução desse indicador ${ }^{(12)}$. Além disso, há que ressaltar que o estado atingiu a meta proposta no ano de $2018^{(10)}$. Nesse sentido, cabe destacar a importância do acesso gratuito e universal aos antirretrovirais e a testagem anti-HIV, no Brasil, inclusive no estado de Santa Catarina, sendo essas medidas descritas, na literatura, como responsáveis pela significativa redução da transmissão vertical e, consequentemente, da incidência da doença em menores de cinco anos ${ }^{(12-13)}$.

Entretanto, é importante refletir também sobre a qualidade da assistência dispensada às mulheres no pré-natal, parto e puerpério, tomando por referência alguns aspectos: o momento do diagnóstico da infecção pelo HIV na mãe, o número de consultas realizadas e a instituição da terapia antirretroviral em tempo hábil ${ }^{(13)}$. Pode-se inferir que todos esses fatores podem ser facilitados com uma melhor cobertura populacional pelas equipes de Atenção Básica.

No entanto, este estudo evidenciou uma diminuição da cobertura populacional, pelas equipes de Atenção Básica, entre os anos de 2017 e 2018. Além disso, o estado não alcançou as metas pactuadas para esse indicador no período estudado ${ }^{(10)}$. Apesar de não existir um parâmetro nacional definido para esse indicador, sendo a meta pactuada pelos estados, a partir de cada realidade epidemiológica, ressalta-se que quanto maior a cobertura populacional pela Atenção Básica maior será o potencial de oferta das ações e serviços de saúde básicos para a população ${ }^{(14)}$. E esse fato pode explicar a correlação significativa e inversa entre o número de casos novos de AIDS em menores de cinco anos de idade e a cobertura populacional pelas equipes de Atenção Básica evidenciadas neste estudo.

Nesse sentido, uma revisão de literatura que teve como objetivo averiguar as dificuldades de mulheres HIV positivas em aderir ao pré-natal na APS, evidenciou que essa adesão ainda é reduzida. $\mathrm{O}$ estudo aponta a adesão ao pré-natal como fator determinante na prevenção da transmissão vertical do HIV e destaca o acesso à APS e à qualidade do pré-natal como fatores significativos para que medidas sejam tomadas, a fim de reduzir essa transmissão(15). Apesar da cobertura populacional pelas equipes de Atenção Básica indicar o acesso à APS, esse indicador mensura a carga horária de trabalho dos profissionais e não o trabalho efetivamente realizado por eles ${ }^{(14)}$. No entanto, aferir a oferta potencial dos serviços de saúde na APS ajuda a compreender o acesso a esse nível de atenção ${ }^{(14-}$ 15). Por isso, a diminuição desse indicador no estado, bem como sua correlação com o aumento do número de casos, em menores de cinco anos de idade, merece discussão, uma vez que a cobertura populacional da Atenção Básica pode aproximar as gestantes das intervenções preventivas capazes de reduzir a transmissão vertical do HIV $^{(5)}$.

Apesar da correlação significativa e inversa entre os indicadores estudados, no estado de Santa Catarina como um todo, os resultados evidenciaram que, no ano de 2018, municípios que apresentaram novos casos de AIDS, em menores de cinco anos de idade, tinham cobertura populacional pelas equipes de atenção básica dentro das metas pactuadas.

Pode-se inferir que, tendo como diretriz a ampliação e qualificação do acesso aos serviços de saúde de qualidade, com ênfase no cuidado humanizado, na equidade e no atendimento das necessidades de saúde ${ }^{(4)}$, uma melhor cobertura populacional pelas equipes de Atenção Básica pode trazer melhores resultados na prevenção da transmissão vertical, mas não deve ser vista, isoladamente. Há evidências de que uma melhor cobertura populacional pelas equipes de Atenção Básica ordena o cuidado no sistema de saúde e favorece sua capacidade resolutiva ${ }^{(16-18)}$, mas, individualmente, pode não ser o suficiente para reduzir a transmissão vertical do HIV.

Destaca-se, nesse sentido, que apesar das ações de promoção e prevenção sobre HIV já estarem difundidas entre as equipes de saúde, o diagnóstico de novos casos (em todas as regiões) e, principalmente, o acompanhamento das pessoas com diagnóstico positivo (em alguns municípios) são processos muito recentes na APS, cercados de polêmica e ainda pouco estudados ${ }^{(19-}$ ${ }^{20)}$. Desse modo, pode-se inferir que estratégias de intervenção devem ser elaboradas na APS, levando-se em consideração os contextos nos quais acontece a transmissão vertical do HIV. 
A avaliação do número de casos novos de AIDS, em menores de cinco anos de idade, fornece subsídios para os processos de planejamento, gestão e avaliação de políticas e ações de saúde direcionadas para o controle da transmissão do HIV/AIDS, em áreas e populações específicas e, principalmente, evidencia os resultados das políticas de prevenção da transmissão vertical. As informações determinadas por esse indicador proporcionam a fundamentação indispensável à avaliação das ações realizadas pelo sistema de saúde ${ }^{(21-22)}$.

Por isso, os resultados encontrados, neste estudo, fortalecem a importância dos processos de cuidado desempenhado pelas equipes de saúde na APS, em especial pela equipe de enfermagem. Sendo a cobertura populacional pelas equipes de Atenção Básica a centralidade da APS e ordenadora do cuidado, cabe ao enfermeiro, como um dos principais agentes moduladores da coordenação do cuidado, fortalecer suas práticas, nesse sentido, garantindo proximidade e acolhimento das gestantes. Uma melhor cobertura populacional, pelas equipes de Atenção Básica assegura a acessibilidade das gestantes aos serviços necessários à prevenção da transmissão vertical e pressupõe uma lógica de organização e funcionamento do sistema de saúde, garantindo vinculação e responsabilização, aspectos fundamentais e inerentes ao trabalho do enfermeiro na APS.

Assim, a gestão terapêutica, o monitoramento da situação de saúde da população e o gerenciamento da equipe de enfermagem e do serviço de saúde são funções essenciais do enfermeiro na APS ${ }^{(23-24)}$ que consolidam a cobertura populacional, melhorando vários aspectos da saúde da população, dentre eles, o cuidado à gestante e a prevenção da transmissão vertical do HIV.

Dentre as limitações deste estudo, destacase que, na utilização de dados secundários, pode haver sub-registros. Essa limitação não reduz a confiabilidade do estudo, mas deve ser considerada na interpretação dos resultados.

\section{CONCLUSÃO}

Este estudo evidenciou um aumento do número de casos novos de AIDS, em menores de cinco anos de idade, no Estado de Santa Catarina, entre os anos de 2017 e 2018, apesar das tendências de declínio desse indicador no Brasil. Apesar do aumento, esse indicador atingiu a meta proposta para o ano de 2018. No período estudado, o estado apresentou diminuição da cobertura populacional pelas equipes de Atenção Básica e esse fato teve associação significativa com o aumento do número de casos novos de AIDS em menores de cinco anos de idade.

Pode-se afirmar que avaliar a dinâmica do número de casos novos, em menores de cinco anos de idade e conhecer os aspectos do cuidado prestado pela APS a ela relacionados é um ponto fundamental para o planejamento de uma gestão de saúde de qualidade. Nesse sentido, o planejamento dos processos de trabalho dos profissionais de saúde pode refletir, diretamente, na população atendida. Desse modo, esse trabalho pode contribuir para o direcionamento da tomada de decisões do enfermeiro referentes à redução do número de casos novos, em menores de cinco anos de idade e reforça a necessidade de práticas relacionadas à prevenção da transmissão vertical, que podem ser facilitadas quando há uma melhor cobertura populacional pelas equipes de Atenção Básica.

Frente aos achados, recomenda-se o fortalecimento das diretrizes institucionais nos municípios estudados, em consonância com as diretrizes do SUS, garantindo cobertura populacional, pelas equipes de Atenção Básica. Recomenda-se que sejam realizados futuros estudos relacionados à identificação das variáveis da qualidade do cuidado na APS preditoras da redução do número de casos novos de AIDS, em menores de cinco anos.

\section{REFERÊNCIAS}

1- Meirelles BHS, Suplici SER, Costa VT, Colaço AD, Forgearini BAO, KuehIKamp VM. Care management: Perspectives from managers, professionals and users of a specialized service facility focused on Human Immunodeficiency Virus/Acquired Immune Deficiency Syndrome. J Nurs Care. 2016;5(5):369. DOI: 10.4172/21671168.1000369

2- Brasil. Ministério da Saúde. Boletim Epidemiológico Especial HIV/Aids. Brasília: Ministério da Saúde; 2019.

3- Brasil. Ministério da Saúde. Portaria de Consolidação $\mathrm{MS} / \mathrm{GM} \mathrm{n}^{\circ}$ 4, de 28 de setembro de 2017. Consolidação das normas sobre os sistemas e os subsistemas do Sistema Único de Saúde. Diário Oficial da União 2017.

4- Conselho de Secretarias Municipais de Saúde de Santa Catarina (COSEMS/SC). Pactuação de Indicadores. Santa Catarina: COSEMS/SC; 2018 [citado em 15 ago 2020]. Disponível em: 
https://www.cosemssc.org.br/pactuacao-de-

\section{indicadores-2018}

5- Miranda AE, Pereira GFM, Araujo MAL, Silveira MF, Tavares LDL, Silva LCF, et al. Avaliação da cascata de cuidado na prevenção da transmissão vertical do HIV no Brasil. Cad Saúde Pública 2016;32(9):1-10. DOI: 10.1590/0102311X00118215

6- Garcia E, Bonelli M, Oliveira A, Clapis M, Leite E. As ações de enfermagem no cuidado à gestante: Um desafio à Atenção Primária de Saúde. J Res Fundam. Care 2018;10(3):863-70. DOI: 10.9789/2175-5361.2018.v10i3.863-870

7- Colaço AD, Meirelles BHS, Heidemann ITSB, Villarinho MV. O cuidado à pessoa que vive com HIV/Aids na Atenção Primária à Saúde. Texto Contexto-Enferm. 2019;28:1-14. DOI: 10.1590/1980-265x-tce-2017-0339

8- Diretoria de Vigilância Epidemiológica do Estado de Santa Catarina. População Residente Estimativas Populacionais - SC - 2013 a 2018 [citado em 15 ago 2020]. Disponível em: http://200.19.223.105/cgi-

bin/dh?popsc/def/popsc.def

9- R Development Core Team. R: A language and environment for statistical computing. Vienna: Foundation for Statistical Computing; 2015.

10- Santa Catarina. Secretaria de Estado de Saúde. Deliberação CIB no 218 de 23 de agosto de 2018. Aprova as metas do rol dos indicadores interfederativos do Estado de Santa Catarina, período de para 2018, conforme Resolução da CIT no 08 de 24 de novembro de 2016, que dispõe sobre o processo de pactuação interfederativa indicadores para o período de 2017 a 2021, de acordo com as prioridades nacionais de saúde. Planilha em anexo. Florianóplis: CIB; 2018 [citado em 12 mar 2020]. Disponível em: https://www.saude.sc.gov.br/index.php/legislaca o/deliberacoes-cib/deliberacoes-2018cib?limit $=20 \&$ limitstart $=100$

11- Segurado AC, Cassenote AJ, Luna EA. Saúde nas metrópoles: Doenças infecciosas. Estud Av. 2016;30(86):29-49. DOI: 10.1590/S010340142016.00100003

12- Traebert J, Silva MF, Nickel DA, Schneider IJC. Estimativa da carga de doença por AIDS em Florianópolis, Santa Catarina, Brasil. Epidemiol Serv Saúde 2015;24(3):517-22. DOI: 10.5123/S1679-49742015000300017

13- Mombelli MA, Barreto MS, Arruda GO, Marcon SS. Epidemia da AIDS em tríplice fronteira: Subsídios para a atuação profissional. Rev Bras
Enferm. 2015;68(3):429-37. DOI: 10.1590/00347167.2015680308i

14- Poças KC, Freitas LRS, Duarte EC. Censo de estrutura da Atenção Primária à Saúde no Brasil: Estimativas de coberturas potenciais. Epidemiol Serv Saúde 2017;26(2):275-84. DOI: 10.5123/s1679-49742017000200005

15- Lima KWS, Antunes JLF, Silva ZP. Percepção dos gestores sobre o uso de indicadores nos serviços de saúde. Saúde Soc. 2015;24(1):61-71. DOI: $10.1590 /$ S0104-12902015000100005

16- Souza SS. Acceso y cobertura en la Atención Primaria a la Salud: Un análisis de los indicadores de tuberculosis en Brasil. Enfermería Comunitaria 2019 [citado em 1 dez 2020]; 15:e12395. Disponível em: http://ciberindex.com/c/ec/e12395

17- Ribeiro TH, Magri CL, Santos AL. Hospitalizações por Diabetes Mellitus em adultos e relação com expansão Atenção Primária no Paraná. Saúde Pesqui. 2019;12(2):323-31. DOI: 10.17765/2176-9206.2019v12n2p323-331

18- Silva LS, Viegas SMF, Nascimento LC, Menezes C, Martins JRT, Potrich T. Universalidade do acesso e acessibilidade no quotidiano da Atenção Primária: Vivências de usuários do SUS. Rev Enferm Cent-Oeste Min. 2020;10:1-9. DOI: 10.19175/recom.v10i0.3575

19- Santos SM, Santos DSS, Bispo TCF, Nunes FN, Silva LGP, Lima SRMP. Transmissão vertical do HIV: Dificuldade na adesão ao pré-natal. Revista Enfermagem Contemporânea 2017;6(1):56-61. DOI: $10.17267 / 2317-3378$ rec.v6i1.1109

20- Melo EA, Maksud I, Agostini R. Cuidado, HIV/Aids e atenção primária no Brasil: Desafio para a atenção no Sistema Único de Saúde. Rev Panam Salud Pública. 2018;42:e151. DOI: 10.26633/RPSP.2018.151

21- Lima KWS, Antunes JLF, Silva ZP. Percepção dos gestores sobre o uso de indicadores nos serviços de saúde. Saúde Soc. 2015;24(1):61-71. DOI: $10.1590 /$ S0104-12902015000100005

22- Albuquerque C, Martins M. Indicadores de desempenho no Sistema Único de Saúde: Uma avaliação dos avanços e lacunas. Saúde Debate 2017;41(nesp):118-37. DOI: 10.1590/010311042017 s10

23- Piovesan G, Paula CC, Lopes LFD, Padoin $S M M$, Kleinubing RE, Silva CB. Qualidade da Atenção Primária na perspectiva de profissionais: Saúde de crianças e adolescentes com HIV. Texto Contexto-Enferm. 2017;26(2): 1-11. DOI: $\underline{10.1590 / 0104-07072017000180016}$ 
24- Ferreira SRS, Périco LAD, Dias VRFG. A complexidade do trabalho do enfermeiro na Atenção Primária à Saúde. Rev Bras Enferm. 2018;71(supl 1):704-9. DOI: 10.1590/0034-71672017-0471

Nota: Este estudo é parte de um macroprojeto que avaliou a cobertura de atenção básica nos municípios de Santa Catarina e o alcance dos indicadores da pactuação interfederativa 2017-2021(4)

Recebido em: $14 / 07 / 2020$

Aprovado em: 04/12/2020

Endereço de correspondência:

Samara Eliane Rabelo Suplici

Rodovia Jornalista Manoel de Menezes, 345, Lagoa da Conceição, Florianópolis, Santa Catarina, Brasil. CEP 88.061700

E-mail: samara.suplici@gmail.com 